\section{Neutrophil function response to aerobic and anaerobic exercise in female judoka and untrained subjects}

\author{
Baruch Wolach, Bareket Falk, Ronit Gavrieli, Einat Kodesh, Alon Eliakim
}

Department of Pediatrics and the Pediatric Hematology Section, Meir General Hospital, Kfar Saba, Israel and the Sackler School of Medicine, Tel Aviv University, Tel Aviv, Israel B Wolach

R Gavrieli

A Eliakim

Ribstein Center for Research and Sport Medicine Sciences, Wingate Institute, Netanya, Israel B Falk

E Kodesh

Correspondence to: Dr A Eliakim, Department of Pediatrics, Meir General Hospital, Kfar Saba 44281, Israel.

Accepted for publication 7 September 1999

\begin{abstract}
Objectives-Recent studies have indicated reduced immunity in trained athletes. Aim-To assess the effects of aerobic and anaerobic exercise on the phagocytic process in 18-26 year old trained female judoka $(n=8)$ and untrained controls $(n=7)$.

Methods-Each subject participated randomly in two different testing sessions (aerobic, 20 minutes of treadmill running at $\mathbf{7 0 - 8 0 \%}$ of maximal heart rate; anaerobic, Wingate anaerobic test). Venous blood samples were drawn before, immediately after, and 24 hours after each session.

Results-There were no significant differences in basal values of net chemotaxis (chemotaxis - random migration), bactericidal activity, and superoxide anion release between the judoka and the untrained women. There was a significant decrease in net chemotaxis 24 hours after the aerobic exercise in both the judoka (from 64 (19) to 39 (13) cells/field, p<0.02) and the untrained controls (from 60 (7) to 47 (12) cells/field, $p<0.05$ ). Bactericidal activity and superoxide anion release did not change significantly after aerobic exercise in either group. There were no significant changes in net chemotaxis, bactericidal activity, and superoxide anion release after anaerobic exercise in either the judoka or untrained women.

Conclusions-The decrease in net chemotaxis after aerobic, but not after anaerobic, exercise, suggests that net chemotaxis is affected by the combination of exercise intensity and duration, and not by the exercise intensity itself. Similar effects of both exercise sessions in the judoka and the untrained women suggest that training had no effect on neutrophil function response to aerobic and anaerobic exercises.

(BrF Sports Med 2000;34:23-28)
\end{abstract}

Keywords: exercise; aerobic; anaerobic; neutrophil function; female athlete

In recent years studies have indicated that intense exercise affects the immune system and may increase susceptibility to infection..$^{1-3}$ Moreover, training during the incubation period of infectious diseases can aggravate the clinical course of the developing disease. ${ }^{45}$

The importance of a normal phagocytic arm for immunological function has been well established. ${ }^{67}$ However, there is little information on the effect of exercise on neutrophil phagocytic activity, especially at different intensities and duration. Studies from our own labo- ratory have recently shown reduced neutrophil function in prepubertal female gymnasts after aerobic exercise compared with sedentary controls, reflecting a possible effect of training level. ${ }^{8}$ However, the contribution of the poor nutritional status that characterised the gymnasts and may affect immune function ${ }^{9-11}$ was not examined. Thus, it is possible that the observed differences in the phagocytic response to aerobic exercise between the gymnasts and untrained girls were partially related to nutritional factors.

The aim of this study was to assess the effects of aerobic exercise on the phagocytic process in well nourished highly trained female judoka in comparison with age and weight matched untrained women. In addition, judo training emphasises anaerobic exercise; therefore, to test a possible training effect after anaerobic exercise, we also assessed neutrophil function after an intense anaerobic exercise task in the two groups.

Based on our previous findings in young girls, ${ }^{8}$ we hypothesised that training level would affect the neutrophil response to exercise, and that an impaired neutrophil response would be more apparent after the aerobic than after the anaerobic exercise task. To the best of our knowledge, the present report describes for the first time the effect of both aerobic and anaerobic exercise in the same subjects and is one of the first studies of highly trained female athletes.

\section{Materials and methods}

Eight healthy postpubertal trained judoka and seven matched healthy controls participated in the study. Puberty was assessed by the criteria developed by Tanner. ${ }^{12}$ The age range of the study participants was $18-26$ years. The judoka (weight 64.1 (8.9) kg; height 165 (8) cm; percentage fat 25.1 (5.6); age 19.9 (1.1)) trained an average of 22 hours a week and participated in national and international level competitions. The control subjects (weight $61.7(3.4) \mathrm{kg}$; height 162 (4) cm; percentage fat 29.5 (4.8), age 22.5 (1.5)) were physically active but did not train regularly in any sport. None of the participants had suffered from any illness for at least two weeks before the study. The purpose of the study was explained to the subjects and all gave their informed consent. The study was approved by the human studies committee at the Meir General Hospital, Kfar Saba, Israel.

In a preliminary visit body height and weight were measured using standard techniques. Body fat was estimated using the sum of four skinfolds (biceps, triceps, subscapular, and suprailiac) as described by Durnin and Womersley. ${ }^{13}$ 
Each subject participated in two different testing sessions (aerobic and anaerobic). The order of the sessions was randomised, and the exercise tests were separated by at least 14 days. Both tests took place in the morning (between 700 and $830 \mathrm{am}$ ). Venous blood (10 $\mathrm{ml}$ ) was withdrawn from an antecubital vein before, immediately after, and 24 hours after each exercise task. In addition, blood was also withdrawn at similar times from a nonexercising control. The judoka trained regularly before the exercise sessions. However, subjects from both groups-that is, controls and judoka - were instructed not to participate in any physical activity during the day before the study, and between the second and third blood withdrawals.

We did not assess the dietary intake of the judoka and untrained subjects in the present study. However, we assume on the basis of recent observations of some of the judoka from this study, as well as of a different group living and training under similar conditions, that there was no apparent deficiency in macronutrients or micronutrients in their diet.

\section{AEROBIC EXERCISE}

Exercise consisted of 20 minutes of treadmill running at a heart rate of $170-180$ beats $/ \mathrm{min}$. This heart rate was chosen as an estimate of $70-80 \%$ of maximal age predicted heart rate. Heart rate was monitored using a Polar Sports Tester (Polar Electro Oy, Finland). Some of the untrained subjects were unable to run for 20 minutes at a heart rate of $170-180$ beats $/ \mathrm{min}$. Therefore, in order for them to complete 20 minutes of exercise, the velocity of the treadmill was reduced during exercise, and they performed a fast walk. Thus untrained girls walked/ran at a velocity of $6-8 \mathrm{~km} / \mathrm{h}$ with a slight slope $(1.5 \%)$, while the judoka ran at a velocity of $10.6-11.3 \mathrm{~km} / \mathrm{h}$ with the same slope $(1.5 \%)$.

\section{ANAEROBIC EXERCISE}

All subjects performed a Wingate anaerobic test on a Fleisch Metabo (Switzerland) cycle ergometer. Resistance was applied according to body mass, with a load of $0.049 \mathrm{~kg} / \mathrm{kg}$. A four to five minute warm up preceded the test, during which time subjects were familiarised with the test procedures and performed two to three maximal unresisted accelerations. The maximal unloaded revolutions per minute were recorded. The Wingate anaerobic test was performed three to four minutes after the warm up. Resistance was applied when subjects reached $95 \%$ of their maximal revolutions per minute. Mean power was the average power attained during the entire 30 second test. The Wingate anaerobic test is known to be a highly reliable and valid test of anaerobic power. ${ }^{14}$

\section{ISOLATION OF POLYMORPHONUCLEAR}

LEUCOCYTES (PMNS)

Human purified PMNs (98\%) were isolated from heparinised venous blood. After sedimentation with 3\% dextran (molecular weight 250 kDa: Sigma, Rehovot, Israel), the leucocyte enriched plasma was layered on to a Ficoll-
Hypaque gradient (Lymphoprep; Nycomed Pharma AS, Oslo, Norway) and centrifuged at $400 \mathrm{~g}$ for 30 minutes, as previously described. ${ }^{15}$ The supernatant was discarded and the pellet subjected to hypotonic lysis for 20 seconds to free the PMNs of contaminating red cells. The PMNs were resuspended for chemotaxis in M199 medium (Earle's salt with L-glutamine; Biological industries, Kibbutz Beth Haemek, Israel). For superoxide anion release, PMNs were suspended in Hanks buffered salt solution (Sigma) and in phosphate buffered saline with $0.2 \%$ glucose and $1 \%$ bovine serum albumin (PBS/GA) for bactericidal activity. Neutrophil functions were assessed in our laboratory as previously reported. $^{6}$

\section{CHEMOTAXIS ASSAY}

A 48 well chemotactic microchamber (Neuro Probe Inc, Bethesda, Maryland, USA) was used to determine random migration and chemotaxis. ${ }^{16}$ Either the chemoattractant $\mathrm{N}$-formylmethionyl-leucyl-phenylalanine (FMLP: Sigma) at a concentration of 10-6 M or the M199 medium was added to the bottom wells. A polycarbonate membrane filter, PVP free, with $3 \mu \mathrm{m}$ pores (Nucleopore Corp, Pleasanton, California, USA) was placed on top of the wells in the bottom plate. The gasket and the top plate were affixed, and $50 \mu \mathrm{l}$ of $10^{6} \mathrm{PMNs} / \mathrm{ml}$ was added to the upper wells. The assembly was incubated for $60 \mathrm{~min}$ in humidified air. After incubation the filter was wiped off and stained with May-GrunwaldGiemsa; the number of cells was determined under light microscopy with a $20 \times$ objective and an optical grid at $10 \times$ magnification. The net chemotaxis was calculated by subtracting the random migration from the chemotactic activity. Cell counting was performed blindly and in duplicate.

\section{BACTERICIDAL ACTIVITY}

Maximal bactericidal activity was quantified as previously reported. ${ }^{17}$ Bacteria (Staphyloccocus aureus; American Type Culture Collection) were freshly grown before each experiment and allowed to enter an early stationary phase (four hours shaking at $37^{\circ} \mathrm{C}$ ). The final concentration of bacteria was determined spectrophotometrically at $640 \mathrm{~nm}$. A suspension of PMNs at a final concentration of $10^{6} / \mathrm{ml}$ was incubated with bacteria at a ratio of 1 to 3 , in the presence of autologous, or a pool of homologous, $\mathrm{AB}$ serum at $37^{\circ} \mathrm{C}$ for 90 minutes, with continuous shaking. The PMNs were then lysed with 5 volumes of distilled water, and the suspensions were diluted and plated $(30 \mu \mathrm{l})$ in triplicate in broth agar plates, for 18 hours at $37^{\circ} \mathrm{C}$. Each experimental set up included two controls comprising PBS/GA and bacteria or autologous serum and bacteria. In these control systems we observed none or less than 0.02 exponential decrease in bactericidal activity respectively $(n>100)$. The colonies were counted and the exponential decrease was calculated and compared with that of the control counts. 


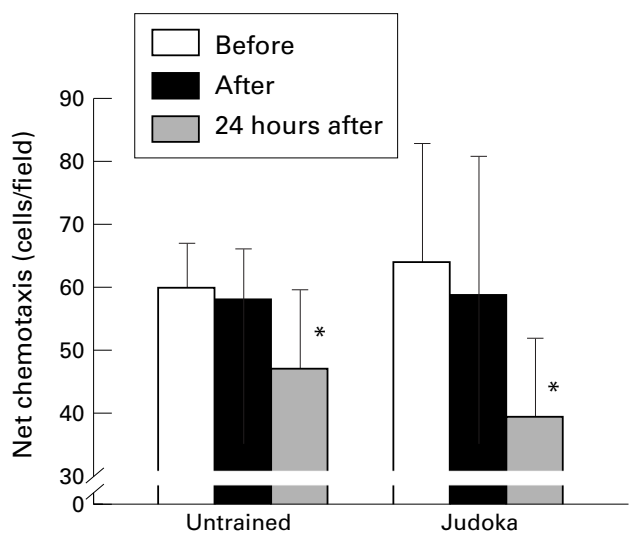

Figure 1 The effect of aerobic exercise on net chemotaxis in judoka and untrained women. There was a significant decrease $\left({ }^{*} p<0.05\right)$ in net chemotaxis in both the judoka and untrained women 24 hours after the aerobic exercise.

SUPEROXIDE ANION RELEASE

This assay was performed as previously reported. ${ }^{18}$ The PMNs were suspended in Hanks buffered salt solution at a final density of $5 \times 10^{6}$ cells $/ \mathrm{ml}$ and incubated at $37^{\circ} \mathrm{C}$ with $60 \mu \mathrm{M}$ ferricytochrome c (Sigma), with or without $214 \mathrm{U}$ superoxide dismutase (Sigma), in a total volume of $1 \mathrm{ml}$. To initiate the reaction, $1 \mu \mathrm{g}$ phorbol 12-myristate 13-acetate (PMA; Sigma) or 0.1 $\mu$ M FMLP (Sigma) was added for 10 minutes in a shaking water bath. The reaction was stopped by placing the tubes in ice and the cells were spun down in a chilled centrifuge. The absorbance of the supernatant was measured at $550 \mathrm{~nm}$. The results of triplicate tests were averaged, and superoxide anion release was calculated using the Massey absorption coefficient of $2.1 \times 10^{4} \mathrm{M}^{-1} \mathrm{~cm}^{-1}$.

\section{STATISTICAL ANALYSIS}

An unpaired $t$ test was performed to assess differences in neutrophil function between the groups before the exercise tests. Variables measured repeatedly-for example,

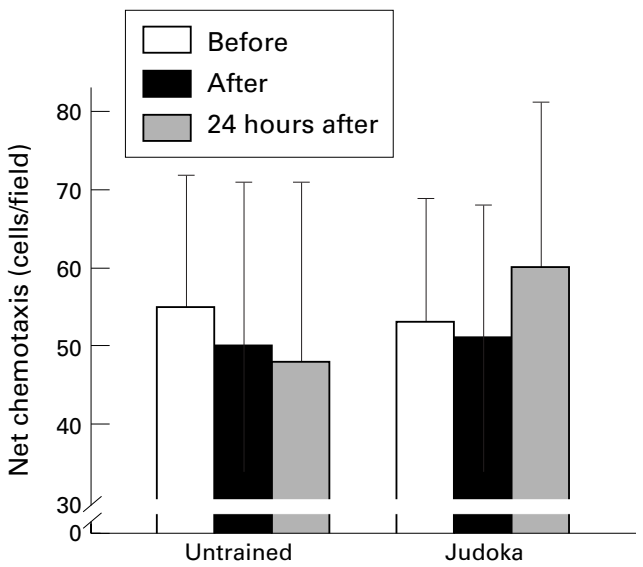

Figure 2 The effect of anaerobic exercise on net chemotaxis in judoka and untrained women. Anaerobic exercise had no effect on net chemotaxis in either.

chemotaxis-were analysed using an analysis of variance for repeated measures, with time serving as the within subject factor and training level as the between subject factor. Post hoc analysis was performed using the Bonferroni procedure. ${ }^{19}$ Statistical significance was set at $\mathrm{p}=0.05$ for all statistical analyses. Data are presented as means (SD).

\section{Results}

AEROBIC EXERCISE

Figure 1 and table 1 summarise the effect of aerobic exercise on neutrophil function. There was a significant decrease in net chemotaxis 24 hours after the aerobic exercise in both judoka and untrained subjects (fig 1). It is important to emphasise that the decrease in net chemotaxis 24 hours after exercise occurred in all the 15 subjects. There were no significant changes in random migration, bactericidal activity with both autologous and homologous serum, and in the PMA and FMLP stimulated superoxide anion release by neutrophils after exercise in either the judoka or untrained controls (table

Table 1 Effect of aerobic exercise on neutrophil function in judoka and untrained women

\begin{tabular}{|c|c|c|c|c|c|c|}
\hline & \multicolumn{3}{|c|}{ fudoka $(n=8)$} & \multicolumn{3}{|c|}{ Untrained $(n=7)$} \\
\hline & Before & After & $24 \mathrm{~h}$ after & Before & After & 24 h after \\
\hline Bactericidal activity- autologous serum & $1.26(0.2)$ & $1.26(0.3)$ & $1.17(0.2)$ & $1.39(0.3)$ & $1.4(0.1)$ & $1.27(0.2)$ \\
\hline Bactericidal activity- homologous serum & $1.15(0.3)$ & $1.1(0.3)$ & $0.96(0.1)$ & $1.23(0.1)$ & $1.18(0.2)$ & $1.14(0.3)$ \\
\hline \multicolumn{7}{|c|}{ PMA induced superoxide anion release $(\mathrm{mmol}$} \\
\hline $\left.\mathrm{O}_{2}^{-} / 10^{6} \mathrm{PMNs} / \mathrm{min}\right)$ & $3.29(0.8)$ & $3.14(1.0)$ & $3.72(0.4)$ & $3.71(0.4)$ & $3.74(0.3)$ & $3.39(0.5)$ \\
\hline $\begin{array}{l}\text { FMLP induced superoxide anion release } \\
\qquad\left(\mathrm{mmol} \mathrm{O}_{2}^{-} / 10^{6} \mathrm{PMNs} / \mathrm{min}\right)\end{array}$ & $1.43(0.4)$ & $1.58(0.5)$ & $2.3(1.3)$ & $1.72(0.9)$ & $1.54(0.5)$ & $1.64(0.3)$ \\
\hline
\end{tabular}

Values are mean (SD). Bactericidal activity is expressed as exponential decrease in number of colonies. PMA, phorbol 12-myristate 13-acetate; FMLP, $N$-formylmethionyl-leucyl-phenylalanine; PMN, polymorphonuclear leucocyte.

Table 2 Effect of anaerobic exercise on neutrophil function in judoka and untrained women

\begin{tabular}{|c|c|c|c|c|c|c|}
\hline \multirow[b]{3}{*}{ Bactericidal activity- autologous serum } & \multicolumn{3}{|c|}{ Fudoka $(n=8)$} & \multicolumn{3}{|c|}{ Untrained $(n=7)$} \\
\hline & Before & After & $24 \mathrm{~h}$ after & Before & After & $24 \mathrm{~h}$ after \\
\hline & $1.08(0.2)$ & $1.13(0.3)$ & $1.2 \quad(0.3)$ & $1.07(0.1)$ & $1.1 \quad(0.2)$ & $1.1 \quad(0.2)$ \\
\hline Bactericidal activity- homologous serum & $1.08(0.2)$ & $1.08(0.2)$ & $1.04(0.3)$ & $1.0 \quad(0.2)$ & $1.2(0.4)$ & $1.04(0.1)$ \\
\hline $\begin{array}{l}\text { PMA induced superoxide anion release } \\
\left(\mathrm{mmol} \mathrm{O}_{2}^{-} / 10^{6} \mathrm{PMNs} / \mathrm{min}\right)\end{array}$ & $3.78(0.5)$ & $3.82(0.3)$ & $3.23(0.8)$ & $3.68(0.4)$ & $3.57(0.59)$ & $3.46(0.5)$ \\
\hline $\begin{array}{l}\text { FMLP induced superoxide anion release } \\
\left(\mathrm{mmol} \mathrm{O}_{2}^{-} / 10^{6} \mathrm{PMNs} / \mathrm{min}\right)\end{array}$ & $2.06(0.6)$ & $1.82(0.6)$ & $2.1 \quad(1.3)$ & $1.41(0.8)$ & $1.32(0.8)$ & $1.55(1.1)$ \\
\hline
\end{tabular}

Values are mean (SD). Bactericidal activity is expressed as exponential decrease in number of colonies. PMA, phorbol 12-myristate 13-acetate; FMLP, $N$-formylmethionyl-leucyl-phenylalanine; PMN, polymorphonuclear leucocytes. 
1). At any time measured there were no significant differences in chemotactic activity, bactericidal activity, and superoxide anion release between the judoka and untrained women.

ANAEROBIC EXERCISE

Figure 2 and table 2 summarise the effect of anaerobic exercise on neutrophil function. There were no significant changes in net chemotaxis (fig 2), bactericidal activity (with both autologous and homologous serum), and superoxide anion release (of both PMA and FMLP stimulated neutrophils) after exercise in either the judoka or untrained controls (table 2 ). There were no significant differences in these variables between groups.

\section{Discussion}

This study examined the effect of an aerobic exercise task compared with a supramaximal anaerobic exercise task on neutrophil function in highly trained judoka and untrained women. At 24 hours after the aerobic exercise there was a significant decrease in neutrophil net chemotaxis in both groups, but not in the neutrophil random migration, bactericidal activity, and superoxide anion release. In contrast, there were no significant changes in neutrophil function after the anaerobic exercise, in either the judoka or untrained controls. The decrease in net chemotaxis after aerobic, but not after anaerobic, exercise suggests that net chemotaxis is affected by the combination of exercise duration and intensity, and not only by the exercise intensity. However, further combinations of exercise intensity and duration need to be examined. In contrast with our hypothesis, similar effects of both exercise sessions in the judoka and untrained women indicate that training had no effect on neutrophil function response to aerobic and anaerobic exercises.

It should be noted that the aerobic exercise was aimed to be an intensity eliciting $70-80 \%$ of the age predicted maximal heart rate. In view of the fact that some of the untrained subjects were unable to run for the full 20 minutes and continued at a fast walk, it is possible that the anaerobic component in this task was higher among this group than in the judokas. Nevertheless, the neutrophil function response after the 20 minute run/walk was similar in both groups.

The decrease in chemotactic activity 24 hours after exercise, which occurred in all the study participants, is consistent with our previous observation of decreased chemotactic activity 24 hours after aerobic exercise in prepubertal female gymnasts. ${ }^{8}$ In contrast, several investigations ${ }^{20-22}$ reported increased chemotactic activity after moderate cycling and treadmill running. Rodriguez et $a l^{23}$ observed no change in chemotaxis among untrained subjects after a short period of running at a similar intensity to that used in this study. This discrepancy suggests that the intensity and duration of exercise may influence the exercise associated chemotactic response. Moreover, the subjects studied in two of the reports ${ }^{20}$ were patients with exercise induced asthma. Therefore the effect of other inflammatory mediators on chemotaxis cannot be ruled out.

The mechanism for the reduced chemotactic activity after exercise is not clearly understood but may involve exercise induced intrinsic changes in the cell itself or may be related to humoral factors which influence chemotaxis. As the circulation half life of PMNs is about seven hours, the reduced chemotaxis found 24 hours after the exercise probably reflects an effect on bone marrow PMNs, presumably of humoral origin. Muns et $a l^{24}$ showed increased neutrophil chemotactic activity of PMNs obtained from nasal lavage of amateur runners immediately after a marathon race. This increased local chemotactic activity may reflect a local immune adaptation to the increased exposure to respiratory pathogens during a long competitive race. Whether this increase in local chemotactic activity contributes to the decrease in systemic chemotactic activity shown in the present study is still speculative.

We did not assess chemotactic activity more than 24 hours after exercise and therefore do not know when it recovers or returns to values found before exercise. However, the normal resting values before the beginning of the physical effort, and the fact that there were no differences in values found before exercise between the groups, suggests complete recovery with time and indicates only transient changes after exercise. The clinical implications of the transient decrease in net chemotaxis after aerobic exercise are still not clear. However, the possibility that this transient decrease in net chemotaxis reflects an "open window" in which the host defence mechanism is decreased and the risk of infection is increased ${ }^{25} 26$ has to be considered.

Bactericidal activity and superoxide anion release were not significantly affected by aerobic or anaerobic exercise in the present study. This is consistent with reports of Benoni et $a l^{2^{7}}$ who showed no effect of 10 minutes of cycle ergometry on cellular adhesion, bactericidal activity, and superoxide anion production in active and inactive subjects. We previously reported, however, a decrease in PMA induced superoxide anion release after the same aerobic exercise protocol in prepubertal female gymnasts compared with untrained girls. ${ }^{8}$ The apparent inconsistency between the present study and our previous study with the gymnasts may be related, at least partially, to the poor nutritional status of the gymnasts ${ }^{1128}$ (as mentioned above, the gymnasts had significantly lower body weight and their energy intake was low). In the present study, the untrained girls had a somewhat higher percentage body fat (insignificant) compared with the judoka, and may be considered overweight. While it is known that malnutrition may affect neutrophil function, the possible effect of being overweight is unknown. Nevertheless, in this study, no differences were observed between the two groups. In addition, the differences in the response to aerobic exercise between 
prepubertal gymnasts and postpubertal judoka may be related to maturational factors.

In contrast, other studies ${ }^{29-31}$ have shown a decrease in bactericidal activity after longer exercise duration - for example, 50 mile walking race $^{29}$ and $100 \mathrm{~km} \mathrm{run}{ }^{30}$-, after graded treadmill exercise to exhaustion, ${ }^{32}$ and during periods of intense training. ${ }^{33}$ This suggests that extensive aerobic exercise and/or intensive training can result in alterations in immune function. The inconsistency in results may reflect differences in exercise intensity, fitness level, or training status of the subjects, as well as differences in gender and nutritional status. Interestingly, the previously reported changes in bactericidal activity after extensive aerobic exercise and/or intensive training correlated with elevated levels of cortisol and ketone bodies ${ }^{29}$ and with levels of adrenaline (epinephrine). ${ }^{33}$ These variables were not assessed in this study.

There were no significant differences in neutrophil function between the judoka and untrained women before the exercise sessions, indicating that intensive training that emphasises mainly anaerobic exercise does not chronically affect neutrophil function. Several studies, however, showed decreased bactericidal activity among elite prepubertal female gymnasts $^{8}$ and trained adult cyclists ${ }^{34}{ }^{35}$ compared with untrained controls. Smith et $a l^{36}$ suggested that partial suppression of neutrophil activity in athletes serves to reduce the inflammatory response to low level tissue damage which may occur during regular training. In addition, the reduced bactericidal activity among elite athletes may reflect reduced immunity resulting from intense physiological and psychological stress. In contrast, Benoni et $a l^{\beta 7}$ reported increased bactericidal activity and superoxide anion release in professional basketball players during the season. The mechanism of these differences is not clearly understood, but may be attributable to differences in age, maturation, gender, nutritional factors, different training methods-for example, aerobic $v$ anaerobic - , the initial fitness level of the subjects studied, as well as different methodological procedures.

In summary, in this study aerobic exercise induced a chemotactic activity in young adult trained and untrained women. This observation supports the concept that exercise may adversely affect the immune system. ${ }^{38} \mathrm{Al}-$ though some studies-for example, that of Pyne et $a l^{39}$ - failed to find an association between reduced neutrophil function and increased incidence of upper respiratory tract infection during training, the interrelation between exercise related immune deficits and the development of infection should be further evaluated. In addition, the practical value of this knowledge for monitoring the health and training programmes of elite athletes requires further clarification.

Contributors: B W participated in formulating the hypothesis and design of the study, interpretation of the results, and writing of the paper. B F participated in formulating the design of the of the paper. B F participated in formulating the design of the
study and data collection, performed the statistical analysis, and participated in writing the paper. R G performed data collection participated in writing the paper. R G performed data collection and analysis of the neutrophil function. E K participated in for-
mulating the study design, recruiting subjects, and execution of the study. A E initiated and coordinated the study hypothesis, participated in designing the study, collecting the data, interpreting the results, and writing the paper.

This study was supported by the Israeli Sports Authority and the Terner Research Fund.

1 Berglund B. Infectious disease in elite cross-country skiers: a one-year incidence study. Clin Sports Med 1990;2:19.

2 Fitzgerald L. Overtraining increases the susceptibility to infection. Int $\mathcal{F}$ Sports Med 1991;12:S5-8.

3 Nieman DC, Johansen LM, Lee JW, et al. Infectious episodes in runners before and after the Los Angeles maraepisodes in runners before and after the Los Ang
thon. $\mathcal{F}$ Sports Med Phys Fitness 1990;30:316-21.

4 Gautmanitan BG, Ghason JL, Lerner AM. Augmentation of Gautmanitan BG, Ghason JL, Lerner AM. Augmentation of
the virulence of murine Coxsackie virus B3 myocardiopathe virulence of murine Coxsackie virus B3

5 Russell WR. Poliomyelitis, the paralytic stage and the effect of physical activity on the severity of paralysis. BMF 1949; $1: 465-71$

6 Wolach B, Baehner RL, Boxer LA. Clinical and laboratory approach to the management of neutrophil dysfunction. Isr F Med Sci 1982;18:897-916.

7 Curnutte JT. Disorders of granulocyte function and granulopoiesis. In: Nathan DG, Oski FA, eds. Hematology of 1993:904-77.

8 Wolach B, Eliakim A, Gavrieli R, et al. Aspects of leukocyte function and the complement system following aerobic exercise in young female gymnasts. Scand $\mathcal{F}$ Med Sci Sports 1998;8:91-7.

9 Schmidt K, Moser U. Vitamin C: a modulator of host defense mechanism: an overview. Int $\mathcal{F}$ Vitam Nutr Res Suppl 1985;27:363-79.

10 Chandra RK, Grace A. Goldsmith Award Lecture. Trace element regulation of immunity and infection. $7 \mathrm{Am}$ Coll Nutr 1985;4:5-16.

11 Harris MC, Douglas SD, Lee JC, et al. Diminished polymorphonuclear leukocyte adherence and chemotaxis following protein-calorie malnutrition in newborn rats. Pediatr Res 1987;21:542-6.

12 Tanner JM. Growth at adolescence. 2nd ed. Oxford: Blackwell Scientific Publications, 1962.

13 Durnin, JVGA, Womersley J. Body fat assessed from total body density and its estimation from skinfold thickness: measurements on 481 men and women aged from 16 to 72 years. Br ₹ Nutr 1974;32:77-97.

14 Inbar O, Bar-Or O, Skinner JS. The Wingate anaerobic test. Champaign, IL: Human Kinetics, 1996

15 Boyum A. Isolation of mononuclear cells and granulocytes from human blood. Scand $\mathcal{F}$ Clin Lab Invest 1968;21 (suppl): $77-89$.

16 Falk W, Goodwin RH Jr, Leonard EJ. A 48 well micro-chemotaxis assembly for rapid and accurate measurement of leukocyte migration. F Immunol Methods 1980;33:239-47.

17 Clawson CC, Repine JE. Quantitation of maximal bactericidal capability in human neutrophils. F Lab Clin Med 1976;88:316-27.

18 Weening RS, Wever R, Roos D. Quantitative aspects of the production of superoxide radicals by phagocytizing human granulocytes. $\mathcal{F}$ Lab Clin Med 1975;85:245-52.

19 Hochberg Y. A sharper Bonferroni procedure for multiple tests of significance. Biometrika 1988;75:800-2.

20 Nagy L. Serum neutrophil chemotactic activity and exercise-induced asthma. Eur F Respir Dis 1983;64:161-5.

21 Lee TH, Nagakura T, Cromwell O, et al. Neutrophil chemotactic activity and histamine in atopic and nonatopic subjects after exercise-induced asthma. Am Rev Respir Dis 1984;129:409-12.

22 Ortega E, Barriga C, De La Fuente M. Increased chemotaxis of neutrophils in untrained subjects after $1 \mathrm{~h}$ of cycling at 50\% $\mathrm{VO}_{2}$ max. Eur F Appl Physiol 1993;66:60-4.

23 Rodriguez AB, Barriga C, De La Fuente M. No changes of chemotaxis after a short period of running at $80 \%$ of the maximum heart rate in untrained subjects. Int $\mathcal{F}$ Sports Med 1991;12:276-80

24 Muns G. Effect of long distance running on polymorphonuclear neutrophil phagocytic function of the upper airways. Int $\mathcal{F}$ Sports Med 1994;15:96-9.

25 Nieman, DC. Exercise, infection and immunity. Int $\mathcal{F}$ Sport Med 1994;15:S131-41.

26 Nieman, DC. Upper respiratory tract infections and exercise. Thorax 1995;50:1229-31.

27 Benoni G, Bellavite P, Adami A, et al. Effect of acute exercise on some hematological parameters and neutrophil
functions in active and inactive subjects. Eur 7 Appl Physiol functions in active

$28 \mathrm{Babu}$ U, Failla ML. Copper status and function of neutrophils are reversibly depressed in marginally and severely copper deficient rats. $\mathscr{F}$ Nutr 1990;120:1700-9.

29 Fukatsu A, Sato N, Shimizu H. 50-mile walking race suppresses neutrophil bactericidal function by inducing increases in cortisol and ketone bodies. Life Sci 1996;58: 2337-43

30 Gabriel H, Muller HJ, Kettler K, et al. Increased phagocytic capacity of the blood, but decreased phagocytic activity per individual circulating neutrophil after an ultra distance run. Eur f Appl Physiol 1995;71:281-4.

31 Pyne DB, Baker MS, Smith JA, et al. Exercise and neutrophil oxidative burst: biological and experimental neutrophil oxidative burst: biological and expe
variability. Eur $\mathcal{F}$ Appl Physiol 1996;74:564-71.

32 Hack V, Strobel G, Ran JP, et al. The effect of maximal exercise on the activity of neutrophil granulocytes in highly 
trained athletes in a moderate training period. Eur $\mathrm{f}$ Appl Physiol 1992;65:520-4.

33 Hack V, Strobel G, Weiss M, et al. PMN cell counts and phagocytic activity of highly trained athletes depend on training period. F Appl Physiol 1994;77:1731-5.

34 Blannin AK, Chatwin LJ, Cave R, et al. Effects of submaximal cycling and long-term endurance training on neutrophil phagocytic activity in middle aged men. $\mathrm{Br} \mathcal{F}$ Sports Med 1996;30:125-9.

35 Lewicki R, Tchorzewski H, Denys A, et al. Effects of physical exercise on some parameters of immunity in conditioned sportsmen. Int f Sports Med 1987;8:309-14.
36 Smith JA, Telford RD, Mason IB, et al. Exercise, training and neutrophil microbicidal activity. Int 7 Sports Med 1990; and neutrop

37 Benoni G, Bellavite P, Adami A, et al. Changes in several neutrophil functions in basketball players before, during and after the sports season. Int $\mathcal{F}$ Sports Med 1995;16:347 .

38 Smith JA, Pyne DB. Exercise, training and neutrophil function. Exerercise and Immunology Reviews 1997;3:96-116.

39 Pyne DB, Baker MS, Fricker PA, et al. Effects of an intensive 12-week training program by elite swimmers on neutrophil oxidative activity. Med Sci Sport Exerc 1995;27:536-42.

\section{Take home message}

Aerobic exercise decreases net chemotactic activity in young trained and untrained women. This supports the concept that exercise may adversely affect the immune system, and awareness of this should be increased among sports physicians.

\section{Fighting spirit}

Fencing is addictive, probably more so than many sports. I started at the age of 10, with bamboo sticks at school in Singapore. At 14, in my first competition, the British Junior Schoolgirls, I reached the semifinal but won the style prize - a cup that outclassed the winner's trophy. That was it, and for the next 13 years I trained hard: technical lessons from my coach, fighting practice at the club, footwork sessions, circuit training for strength, running for stamina and the competition circuit for tactical experience, and those oh so satisfying victories, with a few medals thrown in along the way plus a great social life.

I enjoy the fights: permission to be aggressive in a controlled and safe way against all sorts of male and female opponents. I love the unique combination of physical skill, mental agility, fitness, and competitive toughness that produces winners, and the excitement of defeating a difficult adversary through speed and guile. Individual lessons, with their infinite variety of moves involving hand, arm, feet, body, and brain, demand concentration and hard work from both the fencer and the coach. This special relationship is vital to success in a way that is unique to fencing.

Now, having enjoyed my first international career while a student, first of biochemistry then of medicine, I am competing again with the British Veterans Team, so it's back to the running and the gym. My stamina and reactions gratifyingly improve as I train, and beating the younger fencers annoys them no end.

This time around, although the fencing is serious, the events are sociable, without the emotional extremes and stress of Olympic sport. It's becoming addictive again!

CLARE HALSTED London 